FUNCTION SPACES IX

BANACH CENTER PUBLICATIONS, VOLUME 92

INSTITUTE OF MATHEMATICS

POLISH ACADEMY OF SCIENCES

WARSZAWA 2011

\title{
DIMENSION-INVARIANT SOBOLEV IMBEDDINGS
}

\author{
MIROSLAV KRBEC ${ }^{1}$ \\ Institute of Mathematics, Academy of Sciences of the Czech Republic \\ Žitná 25, 11567 Praha 1, Czech Republic \\ E-mail:krbecm@math.cas.cz \\ HANS-JÜRGEN SCHMEISSER ${ }^{2}$ \\ Mathematisches Institut, Fakultät für Mathematik und Informatik \\ Friedrich-Schiller-Universität \\ Ernst-Abbe-Platz 1-2, 07743 Jena, Germany \\ E-mail:mhj@uni-jena.de
}

Abstract. We survey recent dimension-invariant imbedding theorems for Sobolev spaces.

1. Introduction. Here we will be interested in inequalities

$$
\left(\int_{\Omega}|f(x)|^{p} V(x) d x\right)^{1 / p} \leq c\left(\int_{\Omega}|\nabla f(x)|^{p} d x\right)^{1 / p}, \quad f \in W_{0}^{1, p}(\Omega),
$$

and

$$
\left\|f\left|L^{p} \log ^{\alpha}(1+L)\|\leq c\| \nabla f\right| L^{p}\right\|, \quad f \in W_{0}^{1, p}(\Omega),
$$

where either $\Omega$ is a bounded domain in $\mathbb{R}^{N}$ (specifically, $\Omega$ will be the unit cube in $\mathbb{R}^{N}$ ) or $\Omega=\mathbb{R}^{N}$ and $V$ is a weight on $\Omega$, that is, a.e. non-negative and locally integrable function on $\Omega$. Our concern lies in behaviour of the constant $c$ on the right hand sides - it should be independent not only of $f$ but also of $N$ (and of course it might depend on $V$ ). Both inequalities can naturally be viewed as imbedding theorems independent of the dimension.

2010 Mathematics Subject Classification: Primary 46E35; Secondary 46 E30.

Key words and phrases: Sobolev space, imbedding theorem, uncertainty principle, trace inequality, best constants for imbeddings.

1 The research of this author is carried out in the framework of the Institutional Research Plan No. AV0Z10190503 (Inst. of Math., Acad. Sci. of the Czech Republic). The author also gratefully appreciates the support of the Grant Agency of the Czech Republic under No. 201/06/0400 and the Nečas Center for Mathematical Modeling, LC 06052.

${ }^{2}$ Both authors appreciate support of the DAAD project A/09/04355.

The paper is in final form and no version of it will be published elsewhere. 
Inequality (1.1) has been studied in $\mathbb{R}^{N}$ or on domains in $\mathbb{R}^{N}$ using various methods, in particular, in connection with a boom of weighted inequalities beginning in the 1970s. It has been named the trace inequality or the uncertainty principle. They have found numerous applications in analysis.

As to (1.1) let us recall at least [1], [30], [31], [34], [19], [12], the special case $N=2$ in [13], [26], [2].

The Sobolev imbedding theorem states that $W_{0}^{1, p}(\Omega)(1 \leq p<N, \Omega$ in general with sufficiently smooth boundary) is imbedded into $L^{q}(\Omega)$, where $1 / q=1 / p-1 / N$. Hence if $N \rightarrow \infty$, then $q \rightarrow p_{+}$and one can ask whether there exists some residual improvement of the integrability, that is, a suitable proper subspace of $L^{p}$, into which $W_{0}^{1, p}(\Omega)$ is imbedded and the norm of the imbedding is independent of $N$. It is clear that such an invariant target must be sought outside the range of Lebesgue spaces. Inequality (1.2) turns out to be a good candidate for such a relation. Let us point out that one should be very careful when working with norms - the usual concept of equivalent norms is generally dependent on the dimension without explicitly mentioning it (see also [38], [39] for some observations in this direction).

The question concerning independence of the dimension is also important, for instance, in the theory of contraction semigroups and finds applications in quantum physics (see e.g. [27] for some of the references).

Agreement on notation. Throughout the paper we shall use standard notation for the Sobolev, Lebesgue, Lorentz and Orlicz spaces, the respective domain will be sometimes omitted if no confusion can arise. Sometimes we shall write $\left\|f \mid L^{p}\right\|$ etc. instead of $\|f\|_{p}$ and the like for the sake of better legibility. Since we work with Sobolev spaces of functions with zero traces one point should be emphasized: If $\Omega$ is a domain in $\mathbb{R}^{N}$, then the norm in $W_{0}^{1, p}(\Omega)$, in the Sobolev space of functions with zero traces, will be taken as $\left\|\nabla f \mid L^{p}(\Omega)\right\|$. We define the space $W_{0}^{1, p}(\Omega)$ itself as a completion of $C_{0}^{\infty}(\Omega)$. Note that one should be cautious here: Generally this space does not coincide with the space of functions in $W^{1, p}\left(\mathbb{R}^{N}\right)$ whose support is contained in $\bar{\Omega}$. If, nevertheless, $\Omega$ has a Lipschitz boundary, then both concepts coincide. The latter space is sometimes denoted by $\widetilde{W}^{1, p}(\Omega)$ to mark the difference (see Triebel [37] for a detailed discussion).

Various constants independent of functions will be often denoted by the same symbol $c$ and the like.

If $V$ is a weight in a domain $\Omega \subset \mathbb{R}^{N}$ then the weighted Lebesgue space $L^{p}(V)=$ $L^{p}(V)(\Omega)$ is defined as the space of all measurable $f$ on $\Omega$ with the finite norm $\left\|f \mid L^{p}(V)\right\|=\left(\int_{\Omega}|f(x)|^{p} V(x) d x\right)^{1 / p}$. If $f$ is a measurable function in $\mathbb{R}^{N}$, then $f^{*}$ will denote its non-increasing rearrangement.

If $\Phi$ is a Young function, that is, $\Phi$ is even, convex, $\Phi(0)=0, \lim _{t \rightarrow \infty} \Phi(t) / t=\infty$, and $\Omega \subset \mathbb{R}^{N}$ is measurable, then $m(\Phi, f)=\int_{\Omega} \Phi(f(x)) d x$ is the modular, and the (quasi)norm in the corresponding Orlicz space $L_{\Phi}=L_{\Phi}(\Omega)$ is the Minkowski functional of the modular unit ball, namely, $\left\|f \mid L_{\Phi}\right\|=\inf \{\lambda>0: m(\Phi, f / \lambda) \leq 1\}$ (the Luxemburg norm). Replacing $d x$ by $V(x) d x$ where $V$ is a weight function, we get the weighted Orlicz space $L_{\Phi}(V)$ (or $L_{\Phi}(\Omega, V)$ in a more detailed notation).

One can weaken the assumptions on $\Phi$, for instance $\Phi$ can be just increasing rather 
than convex. In particular, if such a function $\Phi$ is convex on some interval $(a, \infty)(a>0)$ and is equivalent to some convex function on $(0, \infty)$, then we get the same space (with an equivalent norm). If, moreover, $\Omega$ has finite measure and $\Phi$ is convex on some interval $(a, \infty)$, then it is always possible to find an equivalent function which is convex on $(0, \infty)$. We refer to [20] and [33] for the theory of classical Orlicz spaces and of general modular spaces, respectively.

We shall restrict ourselves to a characterization of weighted Orlicz spaces $L_{\Phi}(V)=$ $L_{\Phi}(\Omega, V)$, generated by the modular $m(\Phi, V, f)=\int_{\Omega} \Phi(f(x)) V(x) d x$ as special MusielakOrlicz spaces. Generally, if $\Phi=\Phi(x, t): \Omega \times \mathbb{R} \rightarrow[0, \infty)$ is the generalized Young function or the Musielak-Orlicz function, that is, $\Phi$ is a Young function of the variable $t$ for each fixed $x \in \Omega$ and a measurable function of the variable $x$ for each fixed $t \in \mathbb{R}$, then $m(\Phi, f)=\int_{\Omega} \Phi(x, f(x)) d x$ is a modular and we can consider the corresponding Orlicz space, which is called the Musielak-Orlicz space. Hence with the modular $m(\Phi, V, f)$ the weighted Orlicz spaces becomes a Musielak-Orlicz space.

In the sequel we will work with special Orlicz spaces, usually denoted by $L^{p} \log ^{\alpha}(1+L)$ $(1 \leq p<\infty, \alpha>0)$. The generating function here is $t \mapsto|t|^{p} \log ^{\alpha}(1+|t|), t \in \mathbb{R}$. Further, $L_{\exp t^{\alpha}}$ for $\alpha>0$ will stand for the space with the generating function $t \mapsto$ $\exp \left(|t|^{\alpha}\right)-1, t \in \mathbb{R}$. For $\alpha=1$ we shall simply write $L^{p} \log (1+L)$ and $L_{\text {exp }}$. Note that the function $t \mapsto t^{p} \log ^{\alpha}(1+t)$ is not generally convex near the origin. It is, however, a purely technical problem to consider an equivalent Young function (convex on the whole of $\mathbb{R})$. No confusions can arise so that we use the traditional notation $L^{p} \log ^{\alpha}(1+L)$.

2. Main theorems. Let us summarize the basic claims. Ideas of proofs will be given in the following and in the last section.

First of all we shall make use of the (generalized) Gross logarithmic inequality to get the following

Theorem 2.1 ([23], Theorem 2.3, [24], Theorem 2.3). Let $2 \leq p \leq \infty$. Then

$$
\int_{Q}|f(x)|^{p} \log \left(1+\frac{|f(x)|}{\|\nabla f\|_{p}}\right) d x \leq c\left\|\nabla f \mid L^{p}(\Omega)\right\|^{p}
$$

for all $f \in W_{0}^{1, p}(Q)$ and some $c$ independent of $f$ and $N$.

Next theorem will follow from Theorem 2.1; the sketch of the proof will be given in the end of Section 3 .

THEOREM 2.2 ([23], Theorem 2.3). Under the assumptions of the preceding Theorem, if $V \in L_{\mathrm{exp}}$, then

$$
\int_{Q}|f(x)|^{p} V(x) d x \leq c \int_{Q}|\nabla f(x)|^{p} d x, \quad f \in W_{0}^{1, p}(Q) .
$$

In the last section we will then investigate possibilities to improve the power at the logarithmic function in (2.1). It turns out that one can obtain $\alpha \leq p / 2$ for $1<p<N$ in inequality (1.2). 
Theorem 2.3 ([23], Theorem 3.1, [25], Theorem 4.1). If $1<p<N$, then

$$
\left\|f\left|L^{p} \log ^{\alpha}(1+L)\|\leq c\| \nabla f\right| L^{p}\right\|
$$

for all $f \in W_{0}^{1, p}(Q)$ and some $c$ independent of $f$ and $N$.

Let us observe that the preceding theorem holds for an arbitrary domain $\Omega \subset \mathbb{R}^{N}$ and functions in $\widetilde{W}^{1, p}(\Omega)$.

Similarly as above a weighted consequence of Theorem 2.3 is true (we obtain it also with help of other methods):

Theorem 2.4 ([23], Theorem 3.4). Let $1<p<N$ and let $V$ be a weight function in $Q$. Then there exists a constant $c$ independent of $N$ and $f$ such that

$$
\left\|f\left|L^{p}(V)(Q)\left\|\leq c\left(\left\|V \mid L_{\exp t^{2 / p}}(Q)\right\|\right)^{1 / p}\right\| \nabla f\right| L^{p}\right\|
$$

for every $f \in W_{0}^{1, p}(Q)$.

3. Sketches of proofs, $\boldsymbol{\alpha}=1$. Our first attempt to find the target space for the Sobolev imbedding independent of the dimension goes back to [21], where we used the celebrated Gross logarithmic inequality [14], generalized later in various directions by several authors, see, e.g. [15], [16]. Recall that the Gross logarithmic inequality (see [27] for a detailed account and [3], [5], [10] for further interesting discussions of the topic),

$$
\int_{\mathbb{R}^{N}}|f(x)|^{2} \log \left(\frac{|f(x)|^{2}}{\|f\|_{2}^{2}}\right) d x+N\|f\|_{2}^{2} \leq \frac{1}{\pi} \int_{\mathbb{R}^{N}}|\nabla f(x)|^{2} d x
$$

gives, for a function $f \in W^{1,2}\left(\mathbb{R}^{N}\right)$ and supported in a bounded domain $\Omega \subset \mathbb{R}^{N}$, $\left\|\nabla f \mid L^{2}(\Omega)\right\| \leq 1$, and sufficiently large $N$,

$$
\int_{\Omega}|f(x)|^{2} \log |f(x)| d x \leq \frac{1}{2 \pi} \int_{\Omega}|\nabla f(x)|^{2} d x
$$

(since then at least for large $N$ we have $\log \|f\|_{2} \leq 0$; this follows from the claim on the best constant in the Sobolev imbedding and simple application of Hölder's inequality — see $(3.9))$.

Note that one can formally put 0 in the integral on the left-hand side of (3.1) and (3.2) if $f(x)=0$ (which corresponds well to $\lim _{t \rightarrow 0_{+}} t^{\delta} \log t=0$ for any $\delta>0$ ). The left hand side of (3.2) contains generally both positive and negative values and the estimate says that the final balance of that, containing a logarithmic residue integrability improvement is estimated by a multiple of the $L^{2}$-norm of the gradient. In [21] we have employed the Gross theorem to show that

$$
\int_{B}|f(x)|^{2} \log \left(1+|f(x)| /\|\nabla f\|_{2}\right) d x \leq c\left\|f \mid W_{0}^{1,2}(B)\right\|^{2}
$$

with a constant $c$ independent of $f$ and $N$.

First we will consider inequalities generalizing (3.3), namely,

$$
\int_{\Omega}|f(x)|^{p} \log \left(1+\frac{|f(x)|}{\|\nabla f\|_{p}}\right) d x \leq c\left\|\nabla f \mid L^{p}(\Omega)\right\|^{p}
$$


for $2 \leq p<\infty$ and $f \in W_{0}^{1, p}(\Omega)$, and we will also tackle the weighted dimension-free imbedding of the form

$$
\int_{\Omega}|f(x)|^{p} V(x) d x \leq c\left\|\nabla f \mid L^{p}(\Omega)\right\|^{p},
$$

for $f \in W_{0}^{1, p}(\Omega), 2 \leq p<\infty$ (or $1<p<\infty$ ), $\Omega$ being a bounded domain and/or $\mathbb{R}^{N}$, $c$ depending just on $p$ and $V$. Specifically we will consider $\Omega=Q=(0,1)^{N}$, the unit cube in $\mathbb{R}^{N}$. Conditions for $V$, derived from a variant of Ishii's imbedding theorem for generalized Orlicz-Musielak spaces [17] and [33], will be expressed in terms of suitable exponential integrability of (a multiple of) $V$. Let us point out that in [21] we studied the case of functions living in the unit ball of $\mathbb{R}^{N}$, whose measure tends to zero as $N \rightarrow \infty$.

The generalized form of the Gross inequality for $1<p<\infty$, see Gunson [16], has a little surprising form:

$$
\int_{\mathbb{R}^{N}}|f(x)|^{p} \log (|f(x)|) d x+\gamma_{N, p} \leq \int_{\mathbb{R}^{N}}|\nabla f(x)|^{p} d x
$$

for all $f \in W^{1, p}\left(\mathbb{R}^{N}\right),\|f\|_{p}=1$, with

$$
\gamma_{N, p}=\frac{N}{p}+\frac{N \log \pi}{2 p}+\frac{N \log p}{p^{2}}-\frac{N(p-1) \log (p-1)}{p^{2}}-\frac{1}{p} \log \left(\frac{\Gamma(1+N / 2)}{\Gamma\left(1+N / p^{\prime}\right)}\right),
$$

where $\Gamma$ is the Gamma function and $p^{\prime}=p /(p-1)$.

Substituting $f(x) /\|f\|_{p}$ into (3.5) we get a usual Lebesgue form of the above inequality, namely,

$$
\int_{\mathbb{R}^{N}}|f(x)|^{p} \log \frac{|f(x)|}{\|f\|_{p}} d x+\gamma_{N, p}\|f\|_{p}^{p} \leq \int_{\mathbb{R}^{N}}|\nabla f(x)|^{p} d x .
$$

First we make use of (3.7) to get (2.1).

Step 1. For the constant $\gamma_{N, p}$ from (3.6) let us write

$$
\begin{aligned}
\gamma_{N, p} & =\frac{N}{p}+\frac{N \log \pi}{2 p}+\frac{N \log p}{p^{2}}-\frac{N(p-1) \log (p-1)}{p^{2}}-\frac{1}{p} \log \left(\frac{\Gamma(1+N / 2)}{\Gamma\left(1+N / p^{\prime}\right)}\right) \\
& =T_{1}+T_{2}+T_{3}-T_{4}-T_{5} .
\end{aligned}
$$

We have

$$
T_{1}+T_{2}+T_{3}-T_{4}=\frac{N}{p}\left(1+\frac{\log \pi}{2}+\frac{\log p}{p}-\frac{\log (p-1)}{p^{\prime}}\right)
$$

so that $\left|T_{1}+T_{2}+T_{3}-T_{4}\right| \leq c_{1}(p) N$. With help of Stirling's formula,

$$
\left|T_{5}\right| \sim N\left|\frac{1}{2} \log \frac{N}{2 e}-\frac{1}{p^{\prime}} \log \frac{N}{e p^{\prime}}\right|,
$$

hence $\left|T_{5}\right| \leq c_{2}(p) N \log N$, and we get

$$
\int_{\mathbb{R}^{N}}|f(x)|^{p} \log \frac{|f(x)|}{\|f\|_{p}} d x \leq c(p) N \log N\|f\|_{p}^{p}+\int_{\mathbb{R}^{N}}|\nabla f(x)|^{p} d x,
$$

for all $f \in W^{1, p}\left(\mathbb{R}^{N}\right)$.

The best constant $C$ in the Sobolev imbedding $W_{0}^{1, p} \hookrightarrow L^{q}, 1 \leq p<N, N \geq 3$,

$$
\left(\int_{\mathbb{R}^{N}}|f(x)|^{N p /(N-p)} d x\right)^{(N-p) / N p} \leq C\left\|\nabla f \mid L^{p}\right\|, \quad f \in W^{1, p}\left(\mathbb{R}^{N}\right),
$$


is (see e.g. [36])

$$
C=\sqrt{1 / \pi} \frac{1}{N^{1 / p}}\left(\frac{p-1}{N-p}\right)^{1-1 / p}\left(\frac{\Gamma(N) \Gamma(1+N / 2)}{\Gamma(N / p) \Gamma\left(1+N / p^{\prime}\right)}\right)^{1 / N} .
$$

Calculation using asymptotic properties of the Gamma function shows that $C \sim 1 / N^{1 / 2}$.

Let us denote again by $f$ the extension of $f \in W_{0}^{1, p}(Q)$ by zero to the whole of $\mathbb{R}^{N}$. We get

$$
\left\|f\left|L^{p}(Q)\left\|^{p} \leq \frac{c}{N^{p / 2}}\right\| \nabla f\right| L^{p}\left(\mathbb{R}^{N}\right)\right\|^{p}
$$

and altogether

$$
c(p) N \log N\left\|f\left|L^{p}\left\|^{p} \leq \frac{c(p) \log N}{N^{(p / 2)-1}}\right\| \nabla f\right| L^{p}\right\|^{p} .
$$

If $p>2$, the constant on the right hand side is uniformly bounded with respect to $N$.

Inserting this into (3.8) we get, for $2<p<N$,

$$
\int_{Q}|f(x)|^{p} \log \frac{|f(x)|}{\|f\|_{p}} d x \leq c\left\|\nabla f \mid L^{p}\right\|^{p},
$$

for all $f \in W_{0}^{1, p}(Q)$, with a constant $c$ independent of $f$ and $N$. The same is true for any fixed cube $(a, b)^{N}$ in $\mathbb{R}^{N}$. The case $p=2$ follows easily because $\gamma_{N, 2}>0$ so that the term with this constant can be omitted directly.

Step 2. Now we are able to show that for $N \geq 3$ and $2 \leq p<\infty$ there exists $c$ independent of $N$ such that

$$
\int_{Q}|f(x)|^{p} \log \left(1+\frac{|f(x)|}{\|\nabla f\|_{p}}\right) d x \leq c\left\|\nabla f \mid L^{p}\right\|^{p}
$$

for all $f \in W_{0}^{1, p}(Q)$, and the norm of the imbedding of $W_{0}^{1, p}(Q)$ into $L^{p} \log (1+L)$ is independent of $N$. Let $\left\|\nabla f \mid L^{p}\right\|=1$. The estimate (3.11) follows from (3.10) via the auxiliary inequality

$$
\int_{\mathbb{R}^{N}}\left(|f(x)|+\varepsilon^{2} s_{\varepsilon}(x)\right)^{p} \log \left(1+|f(x)|+\varepsilon^{2} s_{\varepsilon}(x)\right) d x \leq c\left\|\nabla f \mid L^{p}\right\|+\varepsilon c(N)<\infty
$$

with some constant $c$ independent of the dimension $(c(N)$ might depend on $N$ but it is independent on $f$ ) and $\varepsilon$ and with a suitable smooth function $s_{\varepsilon}$ living in $(1+\varepsilon) Q$. The desired inequality (3.11) then follows by virtue of Fatou's lemma, by letting $\varepsilon$ tend to zero.

This part of the proof is rather tedious and long. The idea is to take a smooth $s_{\varepsilon}$ such that $s_{\varepsilon}(x)=1$ if $x \in \bar{Q}, s_{\varepsilon}(x)=0$ if $|x| \notin(1+\varepsilon) Q$, and $\left|\nabla s_{\varepsilon}(x)\right| \leq c(N) / \varepsilon$ on $\overline{(1+\varepsilon) Q} \backslash Q$. A careful calculation yields (3.12). We refer to [24] or to [23].

Finally, for a general $f \in W_{0}^{1, p}(Q), f \neq 0$, note that inequality (3.11) holds for $f(x) /\left(\left\|\nabla f \mid L^{p}\right\|\right)$, that is,

$$
\int_{Q}|f(x)|^{p} \log \left(1+\frac{|f(x)|}{\left\|\nabla f \mid L^{p}\right\|}\right) d x \leq c \int_{Q}|\nabla f(x)|^{p} d x .
$$


Step 3. Now we turn our attention to Theorem 2.2. If $V \in L_{\exp t}(Q)$, then (3.11) will be the starting point to show that there exists $c=c(p, V)>0$ such that

$$
\int_{Q}|f(x)|{ }^{p} V(x) d x \leq c(p, V)\left\|\nabla f \mid L^{p}\right\|^{p}
$$

for all $f \in W_{0}^{1, p}(Q)$. The desired imbedding is the second part of the chain of $W_{0}^{1, p}(Q) \hookrightarrow$ $L^{p} \log (1+L)(Q) \hookrightarrow L^{p}(V)(Q)$; this gives a condition for the weight $V$.

Suppose that $V$ is an integrable function on $Q$ and that there exist $K \geq 1$ and a non-negative function $h \in L^{1}(Q)$ such that

$$
t^{p} V(x) \leq K t^{p} \log (1+t)+h(x), \quad t>0, \quad x \in Q .
$$

Note that the use of such an estimate is inspired by Ishii [17], see also [33]. Then one can show that

$$
\text { if } \int_{Q}\left|f_{k}(x)\right|^{p} \log \left(1+\left|f_{k}(x)\right|\right) d x<\frac{\varepsilon}{4 K}, \quad \text { then } \int_{Q}\left|f_{k}(x)\right|^{p} V(x) d x \leq \varepsilon .
$$

for a given $\varepsilon$ and any sequence $f_{k} \in L^{p} \log (1+L)$.

This involves some technical details for which we refer to [23]. To prove the desired imbedding it is enough to show that

$$
\int_{Q}|g(x)|^{p} V(x) d x \leq c \int_{Q}|g(x)|^{p} \log (1+|g(x)|) d x
$$

for all $g$ such that $\left\|g \mid L^{p} \log (1+L)\right\|=1$; the last equality is equivalent to

$$
\int_{Q}|g(x)|^{p} \log (1+|g(x)|) d x=1 .
$$

Should there exist a sequence $\left(g_{k}\right)$, where $g_{k}$ have the $L^{p} \log (1+L)$ norm equal to 1 , and $B_{k} \rightarrow \infty$ such that

$$
\int_{Q}\left|g_{k}(x)\right|^{p} V(x) d x \geq B_{k} \int_{Q}\left|g_{k}(x)\right|^{p} \log \left(1+\left|g_{k}(x)\right|\right) d x=B_{k},
$$

choose $\varkappa<1$ and a sequence $A_{k} \searrow 0$. Then for large $k$,

$$
\int_{Q} A_{k}^{p}\left|g_{k}(x)\right|^{p} \log \left(1+A_{k}\left|g_{k}(x)\right|\right) d x<\frac{\varkappa}{4 K} .
$$

Then

$$
\int_{Q} A_{k}^{p}\left|g_{k}(x)\right|^{p} V(x) d x \leq \varkappa
$$

and putting $A_{k}=1 / B_{k}^{1 / p}$ we get

$$
\int_{Q}\left|g_{k}(x)\right|^{p} V(x) d x \leq \varkappa B_{k},
$$

which contradicts (3.15). Finally, the condition (3.14) is equivalent to

$$
\sup _{t>0}\left[t V(x) / K-t \log \left(1+t^{1 / p}\right)\right] \leq h(x) / K, \quad x \in Q,
$$

with some integrable function $h$. Hence the left hand side of (3.16) should be integrable over $Q$. If we invoke Young's inequality for complementary Young functions (see e.g. [20]), 
this is guaranteed by integrability of $\Psi(V(x) / K)$, where $\Psi$ is a Young function complementary to $t \mapsto|t| \log \left(1+|t|^{1 / p}\right)$. Plainly $\Psi$ is equivalent to $t \mapsto \exp |t|-1$.

Moreover, the norm inequality (1.2) can be proved, too; for the proof see the end of the last section, where it is done for inequalities with more general $\alpha$.

An analogous claim for the integral with $\log \left(2+|f(x)| /\|\nabla f\|_{p}\right.$ ) (or any $a>1$ in place of 2) can also be proved. Both the modulars give the same space with equivalent norms, nevertheless, an analogue of (3.11) (that is, (2.1)) has to be proved. We refer to [23].

REMARK 3.1. As to an unbounded $\Omega$ a detailed inspection of the proofs above shows that the term $\gamma_{N, p}$ from (3.6) is non-negative if $p \in\left[2, p^{*}\right]$, where $p^{*}$ is the unique solution of the equation $e \pi p^{1 / p}=2(p-1)^{1 / p^{\prime}}$.

4. Sketches of proofs and miscellanea, the case of general $\alpha$. In the first part of this section we discuss various ways how to get weighted estimates of type (1.1). In particular we show several alternative proofs of Theorem 2.4. Then we sketch the proof of Theorem 2.3.

The condition on the weight function $V$ in Theorem 2.2 is derived via another imbedding and a natural question is whether one can get a stronger weighted result by a suitable direct method. We will show that this is indeed the case. Of interest in this connection is also the special choice of weights $V=V(x)$ such that $V^{*}(t)=(\log (1 / t))^{\alpha}$, that is, inequalities of the type

$$
\int_{0}^{1} f^{*}(t)^{p} \log ^{\alpha} \frac{1}{t} d t \leq c \int_{Q}|\nabla f(x)|^{p} d x .
$$

Note that by the Hardy-Littlewood inequality for product of functions the left hand side of (4.1) majorizes $\int_{Q}|f(x)|^{p} V(x) d x$ for such weights $V$. Moreover, the weighted integral on the left can be interpreted as a modular and it is well known (cf. e.g. [6]) that it generates the space $L^{p} \log ^{\alpha}(1+L)$ with a norm equivalent to the standard Luxemburg norm there - this, however, does not automatically imply an inequality generalizing (2.1).

It is not difficult to see that $V \in L^{N / p}$ is a sufficient condition for (1.1) in $\mathbb{R}^{N}$ or in $Q \subset \mathbb{R}^{N}$; it is enough to apply Hölder's inequality with conjugate exponents $N /(N-p)$ and $N / p$ to the left hand side of (1.1). This can be slightly sharpened: Since $W_{0}^{1, p}(Q)$ is imbedded into the Lorentz space $L^{N p /(N-p), p}(Q)$ we have, using the Hardy-Littlewood rearrangement inequality,

$$
\begin{aligned}
\int_{Q}|f(x)|^{p} V(x) d x & \leq \int_{0}^{1}\left(f^{*}(t)\right)^{p} V^{*}(t) d t=\int_{0}^{1} t^{(N-p) / N} f^{*}(t)^{p} t^{p / N} V^{*}(t) \frac{d t}{t} \\
& \leq \sup _{0<s<1} s^{p / N} V^{*}(s) \int_{0}^{1}\left(t^{(N-p) / N p} f^{*}(t)\right)^{p} \frac{d t}{t} \\
& =\left\|V\left|L^{N / p, \infty}\|\| f\right| L^{N p /(N-p), p}\right\|^{p} \leq c(N)\left\|V\left|L^{N / p, \infty}\|\| \nabla f\right| L^{p}\right\|^{p},
\end{aligned}
$$

where $c(N)$ is the best constant for the imbedding of $W^{1, p}\left(\mathbb{R}^{N}\right) \hookrightarrow L^{N p /(N-p), p}$. Hence (1.1) holds in $Q \subset \mathbb{R}^{N}$ if $V \in L^{N / p, \infty}(Q)$, similarly in $\mathbb{R}^{N}$. The behaviour of the constant $c(N)$ is known thanks to Alvino [4]: a bit surprisingly the best constant for the refined Sobolev imbedding into $L^{N p /(N-p), p}\left(\mathbb{R}^{N}\right)$ behaves in the same way with respect 
to $N \rightarrow \infty$ as the best constant in (3.9). Consequently

$$
\begin{aligned}
\int_{Q}|f(x)|^{p} V(x) d x & \leq c \frac{\left\|V \mid L^{N / p, \infty}\right\|}{N^{p / 2}}\left\|\nabla f\left|L^{p}\left\|^{p} \leq c \frac{\left\|V \mid L^{N / p}\right\|}{N^{p / 2}}\right\| \nabla f\right| L^{p}\right\|^{p} \\
& \leq c c(p) \frac{\left\|V \mid L^{N / p}\right\|}{(N / p)^{p / 2}}\left\|\nabla f\left|L^{p}\left\|^{p} \leq c c(p) \sup _{q \geq 1} \frac{\left\|V \mid L^{q}\right\|}{q^{p / 2}}\right\| \nabla f\right| L^{p}\right\|^{p} .
\end{aligned}
$$

Let us recall the standard extrapolation fact that

$$
\left\|V \mid L_{\exp t^{\beta}}(Q)\right\| \sim \sup _{q \geq 1} \frac{\left\|V \mid L^{q}(Q)\right\|}{q^{1 / \beta}}<\infty .
$$

The equivalence of norms here is independent of $N$; indeed, one can pass to rearrangements of $V$ in (4.3) and to use the fact that the (one-dimensional) norms of $V^{*}$ are equal to the corresponding norms of $V$. For more on extrapolation conditions using $L^{q}$ and $L^{q, \infty}$ norms see, e.g. [9] or [32]. Hence we have proved Theorem 2.4.

An analogue of Theorem 2.4 holds in $\mathbb{R}^{N}$ (and similarly on sets with an infinite measure). As to a characterization as in (4.3) one has to be careful. In (4.2) we require in fact that

$$
\sup _{q \geq N / p} \frac{\left\|V \mid L^{q}\left(\mathbb{R}^{N}\right)\right\|}{q^{p / 2}}<\infty,
$$

which in contrast to the situation in $Q$ is not equivalent to finiteness of $\sup _{q \geq 1}$.

A remedy is to use Orlicz spaces $E_{r, \beta}\left(\mathbb{R}^{N}\right)$ generated by the Young function $t \mapsto$ $|t|^{r}\left(\exp t^{\beta}-1\right)(\beta>0)$, as suggested in [11] and investigated in details in [35]. The Luxemburg norm in $E_{r, \beta}\left(\mathbb{R}^{N}\right)$ is equivalent to the (extrapolation) norm

$$
\sup _{q \geq r} \frac{\left\|V \mid L^{q}\left(\mathbb{R}^{N}\right)\right\|}{q^{1 / \beta}}
$$

and the constants of the equivalence are independent of $N$.

We get the following variation on Theorem 2.4.

Theorem 4.1 ([23], Theorem 3.5, [25], Theorem 3.2). Let $1<p<N$ and let $V$ be a weight function in $\mathbb{R}^{N}$. Then there exists a constant $c$ independent of $N$ and $f$ such that

$$
\left\|f\left|L^{p}(V)\left(\mathbb{R}^{N}\right)\left\|\leq c\left(\left\|V \mid E_{N / p, p / 2}\left(\mathbb{R}^{N}\right)\right\|\right)^{1 / p}\right\| \nabla f\right| L^{p}\left(\mathbb{R}^{N}\right)\right\|
$$

for every $f \in W^{1, p}\left(\mathbb{R}^{N}\right)$.

An analogous statement holds for a domain $\Omega \subset \mathbb{R}^{N}$ and functions in $\widetilde{W}^{1, p}(\Omega)$.

For the special choice of the weight function $V$ on $Q$, when $V^{*}(t)=(\log (1 / t))^{\alpha}$, Theorem 2.4 yields $\alpha \leq p / 2$. Indeed, since

$$
\sup _{0<s<1} s^{p / N} V^{*}(s)=e^{-\alpha}\left(\frac{\alpha N}{p}\right)^{\alpha}=c N^{\alpha},
$$

we have,

$$
\int_{0}^{1} f^{*}(t)^{p} \log ^{\alpha} \frac{1}{t} d t \leq c\left\|\left.\nabla f\right|^{p}\right\|, \quad f \in W_{0}^{1, p}(Q)
$$

where $\alpha \leq p / 2$. 
Inequalities of the type

$$
\int_{0}^{1} f^{*}(t)^{p} \log ^{\alpha} \frac{1}{t} d t \leq c\left(\left\|\nabla f\left|L^{p}(Q)\left\|^{p}+\right\| f\right| L^{p}(Q)\right\|^{p}\right)
$$

have been recently studied by Martín and Milman [29]. Such relations are of interest because the expression on the left hand side leads to an equivalent norm in $L^{p} \log ^{\alpha}(1+L)$, say, on the unit cube, nevertheless, its dimension-free relations to the usual modular (corresponding to the Young function $|t|^{p} \log ^{\alpha}(1+|t|)$ ) are not immediately clear. In particular, in some response to [22], which handled dimension-free imbeddings of Sobolev spaces on a unit ball, Triebel [38] raised a question about inequalities of the above type for spaces on a unit cube and the dependence of $c$ on the dimension. The answer was given in [29, subsect. 7.1], giving $\alpha=p / 2$ as the "best" exponent in (4.4). The proof is based on the isoperimetric inequality.

Note that the dimension-free estimate (4.4) with $\alpha=p / 2$ is contained in Theorem 2.4. It is not, however, necessary to use an additional technique as in [29] (isoperimetric theorem) or here in this section (extrapolation). A simple short proof can be given: For $f \in W_{0}^{1, p}(Q)$ we have

$$
\begin{aligned}
& \int_{0}^{1} f^{*}(t)^{p}\left(\log \frac{1}{t}\right)^{\alpha} d t \\
& \leq\left(\int_{0}^{1} f^{*}(t)^{N p /(N-p)} d t\right)^{(N-p) / N}\left(\int_{0}^{1}\left(\log \frac{1}{t}\right)^{N \alpha / p} d t\right)^{p / N} \\
& \leq \frac{c}{N^{p / 2}}\left\|\nabla f \mid L^{p}\right\|^{p}\left[\Gamma\left(1+\frac{N \alpha}{p}\right)\right]^{p / N} \\
& \leq \frac{c}{N^{p / 2}}\left\|\nabla f \mid L^{p}\right\|^{p}\left[\left(\frac{N \alpha}{p}\right)^{p /(N \alpha)}\right]^{\alpha}\left(\left[\Gamma\left(\frac{N \alpha}{p}\right)\right]^{p /(N \alpha)}\right)^{\alpha} \\
& \leq \frac{c}{N^{p / 2}}\left\|\nabla f \mid L^{p}\right\|^{p}\left(\frac{N \alpha}{p}\right)^{\alpha} .
\end{aligned}
$$

We have used the claim on the best constant for the Sobolev imbedding and properties of the Gamma function $\left(\Gamma(\xi)^{1 / \xi} \sim \xi\right.$ as $\left.\xi \rightarrow \infty\right)$. Once more we recover the condition $\alpha \leq p / 2$ for the independence of $N$.

In the remainder of the paper we sketch the proof of Theorem 2.3. This is in fact an alternative approach to (1.1) and (1.2) based on extrapolation of standard Sobolev imbedding theorem and knowledge of the best constant (the norm of the imbedding) to overcome limitations given by the form of the Gross inequality. In the first part of this section we have directly proved weighted inequalities with $\alpha=p / 2$ at the logarithmic function. It is, however, impossible to establish better integrability of $f$ from this.

Step 1. Assume that $\alpha>0$. Hölder's inequality and the standard imbedding give, similarly as in Section 3,

$$
\begin{aligned}
& \left(\int_{\Omega}|f(x)|^{p} \log ^{\alpha}(1+|f(x)|) d x\right)^{1 / p} \\
& \quad \leq \frac{c}{N^{1 / 2}}\left\|\nabla f \mid L^{p}\right\|\left(\int_{\Omega} \log ^{N \alpha / p}(1+|f(x)|) d x\right)^{1 / N} .
\end{aligned}
$$


By the inequality $\log (1+\xi) \leq \frac{1}{\varepsilon} \xi^{\varepsilon}, \xi>0, \varepsilon \in(0,1]$, we obtain

$$
\left(\int_{\Omega} \log ^{N \alpha / p}(1+|f(x)|) d x\right)^{1 / N} \leq\left(\frac{1}{\varepsilon}\right)^{\alpha / p}\left(\int_{\Omega}|f(x)|^{N \alpha \varepsilon / p} d x\right)^{1 / N} .
$$

Plainly we have to make the choice $\frac{N \alpha \varepsilon}{p}=\frac{N p}{N-p}$, in other terms,

$$
\varepsilon=\frac{p^{2}}{\alpha(N-p)} \text {. }
$$

By the Sobolev inequality and (4.6),

$$
\begin{aligned}
\left(\int_{\Omega} \log ^{N \alpha / p}(1+|f(x)|) d x\right)^{1 / N} & \leq\left(\frac{1}{\varepsilon}\right)^{\alpha / p}\left(\int_{\Omega}|f(x)|^{N \alpha \varepsilon / p} d x\right)^{1 / N} \\
& \leq\left(\frac{\alpha(N-p)}{p^{2}}\right)^{\alpha / p}\left(\int_{\Omega}|f(x)|^{N p /(N-p)} d x\right)^{1 / N} \\
& \leq\left(\frac{\alpha(N-p)}{p^{2}}\right)^{\alpha / p}\left(\frac{c}{N^{1 / 2}}\right)^{p /(N-p)}\left\|\nabla f \mid L^{p}\right\|^{p /(N-p)}
\end{aligned}
$$

Together with (4.5),

$$
\begin{aligned}
& \left(\int_{\Omega}|f(x)|^{p} \log ^{\alpha}(1+|f(x)|) d x\right)^{1 / p} \\
& \leq \frac{c}{N^{1 / 2}}\left(\frac{\alpha(N-p)}{p^{2}}\right)^{\alpha / p}\left(\frac{1}{N^{1 / 2}}\right)^{p /(N-p)}\left\|\nabla f \mid L^{p}\right\|^{1+p /(N-p)} \\
& \leq c \frac{N^{\alpha / p}}{N^{1 / 2}}\left\|\nabla f \mid L^{p}\right\|^{1+p /(N-p)} .
\end{aligned}
$$

Independence of the right hand side with respect to $N$ is guaranteed by $\alpha \leq p / 2$.

Step 2. Now let $\alpha \leq p / 2$ and $\left\|\nabla f \mid L^{p}\right\|=1 / c^{(N-p) / N}$ with $c$ from the third line in (4.7). Then

$$
\int_{\Omega}|f(x)|^{p} \log ^{\alpha}(1+|f(x)|) d x \leq 1
$$

For an arbitrary $0 \neq f \in W_{0}^{1, p}$ insert $f /\left(c^{(N-p) / N}\left\|\nabla f \mid L^{p}\right\|\right)$ into (4.8). We get

$$
\int_{\Omega} \frac{|f(x)|^{p}}{c^{p(N-p) / N}\left\|\nabla f \mid L^{p}\right\|^{p}} \log ^{\alpha}\left(1+\frac{|f(x)|}{c^{(N-p) / N}\left\|\nabla f \mid L^{p}\right\|}\right) d x \leq 1,
$$

which gives (according to the definition of the Luxemburg norm)

$$
\left\|f\left|L^{p} \log ^{\alpha}(1+L)\left\|\leq c^{(N-p) / N}\right\| \nabla f\right| L^{p}\right\| \leq c\left\|\nabla f \mid L^{p}\right\| .
$$

REMARK 4.2. Now it is enough to mimic arguments from Section 3 that we used in Step 3 to prove (3.13). We get the weighted estimates in Theorem 2.4 again.

\section{References}

[1] D. R. Adams, Traces of potentials arising from translation invariant operators, Ann. Scuola Norm. Sup. Pisa Cl. Sci. (3) 25 (1971), 203-217.

[2] R. A. Adams, General logarithmic Sobolev inequalities and Orlicz imbeddings, J. Funct. Anal. 34 (1979), 292-303. 
[3] R. A. Adams, F. H. Clarke, Gross's logarithmic Sobolev inequality: a simple proof, Amer. J. Math. 101 (1979), 1265-1269.

[4] A. Alvino, Sulla diseguaglianza di Sobolev in spazi di Lorentz, Boll. Un. Mat. Ital. A (5) 14 (1977), 148-156.

[5] W. Beckner, Geometric asymptotics and the logarithmic Sobolev inequality, Forum Math. 11 (1999), 105-137.

[6] C. Bennett, R. Sharpley, Interpolation of Operators, Pure Appl. Math. 129, Academic Press, Boston, 1988.

[7] H. Brézis, S. Wainger, A note on limiting cases of Sobolev embeddings and convolution inequalities, Comm. Partial Differential Equations 5 (1980), 773-789.

[8] F. Chiarenza, M. Frasca, A remark on a paper by C. Fefferman [12], Proc. Amer. Math. Soc. 108 (1990), 407-409.

[9] D. Cruz-Uribe, M. Krbec, Localization and extrapolation in Lorentz-Orlicz spaces, in: Function Spaces, Interpolation Theory and Related Topics (Lund, 2000), de Gruyter, Berlin, 2002, 273-283.

[10] M. Del Pino, J. Dolbeault, The optimal Euclidean $L^{p}$-Sobolev logarithmic inequality, J. Funct. Anal. 197 (2003), 151-161.

[11] D. E. Edmunds, R. M. Edmunds, H. Triebel, Entropy numbers of embeddings of fractional Besov-Sobolev spaces in Orlicz spaces, J. London Math. Soc. (2) 35 (1987), 121-134.

[12] C. L. Fefferman, The uncertainty principle, Bull. Amer. Math. Soc. (N.S.) 9 (1983), 129-206.

[13] J.-P. Gossez, A. Loulit, A note on two notions of unique continuation, Bull. Soc. Math. Belg. Sér. B 45 (1993), 257-268.

[14] L. Gross, Logarithmic Sobolev inequalities, Amer. J. Math. 97 (1975), 1061-1083.

[15] F. Güngör, J. Gunson, A note on the proof by Adams and Clarke of Gross's logarithmic inequality, Appl. Anal. 59 (1995), 201-206.

[16] J. Gunson, Inequalities in Mathematical Physics, in: Inequalities (Birmingham, 1987), Lect. Notes in Pure and Appl. Math. 129, Dekker, New York, 1991, 53-79.

[17] J. Ishii, On equivalence of modular function spaces, Proc. Japan Acad. 35 (1959), 551-556.

[18] T. Iwaniec, A. Verde, On the operator $\mathcal{L}(f)=f \log |f|$, J. Funct. Anal. 169 (1999), 391-420.

[19] R. Kerman, E. Sawyer, The trace inequality and eigenvalue estimates for Schrödinger operators, Ann. Inst. Fourier (Grenoble) 36 (1986), 207-228.

[20] M. A. Krasnosel'skii, Ya. B. Rutitskii, Convex Functions and Orlicz Spaces, Noordhoff, Groningen, 1961.

[21] M. Krbec, H.-J. Schmeisser, A limiting case of the uncertainty principle, in: Proceedings of Equadiff 11, Proceedings of minisymposia and contributed talks (Bratislava, 2005), Bratislava, 2007, 181-187.

[22] M. Krbec, H.-J. Schmeisser, Dimension-free imbeddings of Sobolev spaces, preprint, Prague, 2008.

[23] M. Krbec, H.-J. Schmeisser, Dimension-invariant imbeddings of Sobolev spaces, preprint, Math/Inf/01/10, Jena, 2010.

[24] M. Krbec, H.-J. Schmeisser, On dimension-free Sobolev imbeddings I, DOI: 10.1016/j.jmaa.2011.08.061, J. Math. Anal. Appl. 387 (2012), 114-125.

[25] M. Krbec, H.-J. Schmeisser, On dimension-free Sobolev imbeddings II, DOI: 10.1007/s13163-011-0068-5, Rev. Mat. Complut., to appear. 
[26] M. Krbec, T. Schott, Superposition of imbeddings and Fefferman's inequality, Boll. Unione Mat. Ital. Sez. B Artic. Ric. Mat. (8) 2 (1999), 629-637.

[27] E. H. Lieb, M. Loss, Analysis, second edition, Grad. Stud. Math. 14, Amer. Math. Soc., Providence, RI, 2001.

[28] J. Martín, M. Milman, Isoperimetry and symmetrization for logarithmic Sobolev inequalities, J. Funct. Anal. 256 (2009), 149-178.

[29] J. Martín, M. Milman, Pointwise symmetrization inequalities for Sobolev functions and applications, Adv. Math. 225 (2010), 121-199.

[30] V. G. Maz'ya, Classes of domains and embedding theorems for functional spaces (in Russian), Dokl. Akad. Nauk SSSR 133 (1960), 527-530; English transl.: Soviet Math. Dokl. 1 (1960), 882-885.

[31] V. G. Maz'ya, On the theory of the higher-dimensional Schrödinger operator (in Russian), Izv. Akad. Nauk SSSR Ser. Matem. 28 (1964), 1145-1172.

[32] M. Milman, Extrapolation and Optimal Decompositions with Applications to Analysis, Lecture Notes in Math. 1580, Springer, Berlin, 1994.

[33] J. Musielak, Orlicz Spaces and Modular Spaces, Lecture Notes in Math. 1034, Springer, Berlin, 1983.

[34] E. T. Sawyer, A characterization of two weight norm inequalities for fractional and Poisson integrals, Trans. Amer. Math. Soc. 308 (1988), 533-545.

[35] H.-J. Schmeisser, W. Sickel, Traces, Gagliardo-Nirenberg inequalities and Sobolev type embeddings for vector-valued function spaces, Jenaer Schriften zur Mathematik und Informatik, Math/Inf/24/01, Jena, 2001.

[36] G. Talenti, Best constant in Sobolev inequality, Ann. Mat. Pura Appl. (4) 110 (1976), 353-372.

[37] H. Triebel, Theory of Function Spaces III, Monogr. Math. 100, Birkhäuser, Basel, 2006.

[38] H. Triebel, Tractable embeddings of Besov spaces into Zygmund spaces, preprint, Jena, 2009.

[39] H. Triebel, Tractable embeddings of Besov spaces into Zygmund spaces, this volume, 361-377. 
